\title{
Real-time determination and monitoring of the auroral electrojet boundaries
}

\author{
Magnar Gullikstad Johnsen* \\ Tromsø Geophysical Observatory (TGO), University of Tromsø, N-9037 Tromsø, Norway \\ *Corresponding author: Magnar.G.Johnsen@uit.no
}

Received 31 January 2013 / Accepted 23 June 2013

\begin{abstract}
A method for nowcasting of the auroral electrojet location from real-time geomagnetic data in the European sector is presented. Along the auroral ovals strong electrojet currents are flowing. The variation in the geomagnetic field caused by these auroral electrojets is observed on a routine basis at high latitudes using ground-based magnetometers. From latitude profiles of the vertical component of these variations it is possible to identify the boundaries of the electrojets. Using realtime data from ground magnetometer chains is the only existing method for continuous monitoring and nowcasting of the location and strength of the auroral electrojets in a given sector. This is an important aspect of any space weather programme. The method for obtaining the electrojet boundaries is described and assessed in a controlled environment using modelling. Furthermore a provisional, real-time electrojet tracker for the European sector based on data from the Tromsø Geophyiscal Observatory magnetometer chain is presented. The relationship between the electrojet and the diffuse auroral oval is discussed, and it is concluded that although there may exist time-dependent differences in boundary locations, there exists a general coincidence. Furthermore, it is pointed out that knowledge about the latitudinal location of the geomagnetic activity, that is the electrojets, is more critical for space weather sensitive, groundbased technology than the location of the aurora.
\end{abstract}

Key words. ionosphere (auroral) - aurora - space weather - aeronomy - geomagnetism

\section{Introduction}

The auroral ovals are oval-shaped bands of aurora with radii of approximately $3500 \mathrm{~km}$ and foci in the vicinity of the geomagnetic poles. The ovals represent the regions of Earth's upper atmosphere where electrons and protons are able to precipitate along the geomagnetic field from the near geospace. A picture of the auroral oval as seen from the Dynamic Explorer 1 spacecraft is shown in Figure 1 (left). The auroral ovals are fixed with respect to the sun and the Earth rotates under them. Because of the offset of the geomagnetic dipole axis (and hence the oval centres) with respect to the Earth rotational axis this allows for diurnal changes in the location of the aurora. Under normal, quiet conditions in the European sector, the aurora can be seen over Svalbard during the day and above northern Fennoscandia during the night. Under disturbed conditions the ovals expand and the aurora reaches latitudes where it is not commonly seen. This is illustrated in Figure 1 (right), where aurora can be seen to cover a wide belt from southern Fennoscandia to central Europe.

Since the magnetic field lines inside the polar caps (auroral ovals) are the only field lines with direct access to the solar wind, the area of the polar cap is a direct measure of how much open flux there is in the magnetosphere (cf. Milan et al. 2003, 2007). One may use the area of the polar cap as a measure of the amount of energy stored in the magnetosphere owing to the interaction between the interplanetary medium and the geomagnetic field. The amount of open magnetic flux is governed by the balance of flux erosion on the magnetopause through magnetic reconnection (merging) and open flux destruction through magnetic reconnection in the magnetotail. In a case of unbalanced reconnection where the reconnection on the magnetopause is higher than in the tail, the amount of open flux will increase and consequently the size of the polar cap will increase moving the auroral ovals equatorwards. In the opposite case where reconnection is more efficient in the magnetotail, the polar cap will shrink moving the auroral ovals polewards. By monitoring the polar cap area and the change in it, it is possible to assess the balance between opening and closure of magnetic flux and to derive total reconnection rate in the magnetospheric system (Milan et al. 2003); this is important in the study of magnetic storms and substorm processes. Furthermore, by monitoring the instantaneous change in the size and shape of the polar cap, by identifying the auroral oval and its movements, the temporal and spatial nature and variations of reconnection at the magnetopause and in the magnetotail may be studied. On a global scale, the shape of the auroral oval may reveal the topology and nature of the magnetopause reconnection $x$-line and effects on the magnetosphere from solar wind parameters such as the dynamic pressure and IMF orientation (e.g., Elphinstone et al. 1990; Kabin et al. 2004; Rae et al. 2010). Thus, the nowcasting and forecasting of the location and size of the auroral ovals have great relevance to Space Weather monitoring.

Several studies have aimed to establish methods for nowcasting and forecasting of the location of the auroral ovals. The OVATION Prime empirical model has been developed using energetic particle measurements from the polar-orbiting Defence Meteorological Satellite Program (DMSP) satellites in order to determine where the auroral oval is and how strong the aurora is (Newell et al. 2002, 2010a, 2010b; Machol et al. 2012). It is currently, as of January 2013, available through several different web portals: The NOAA OVATION auroral forecast (http:/helios.swpc.noaa.gov/ovation/ and http://www.ngdc.noaa.gov/stp/ovation_prime/), which uses 


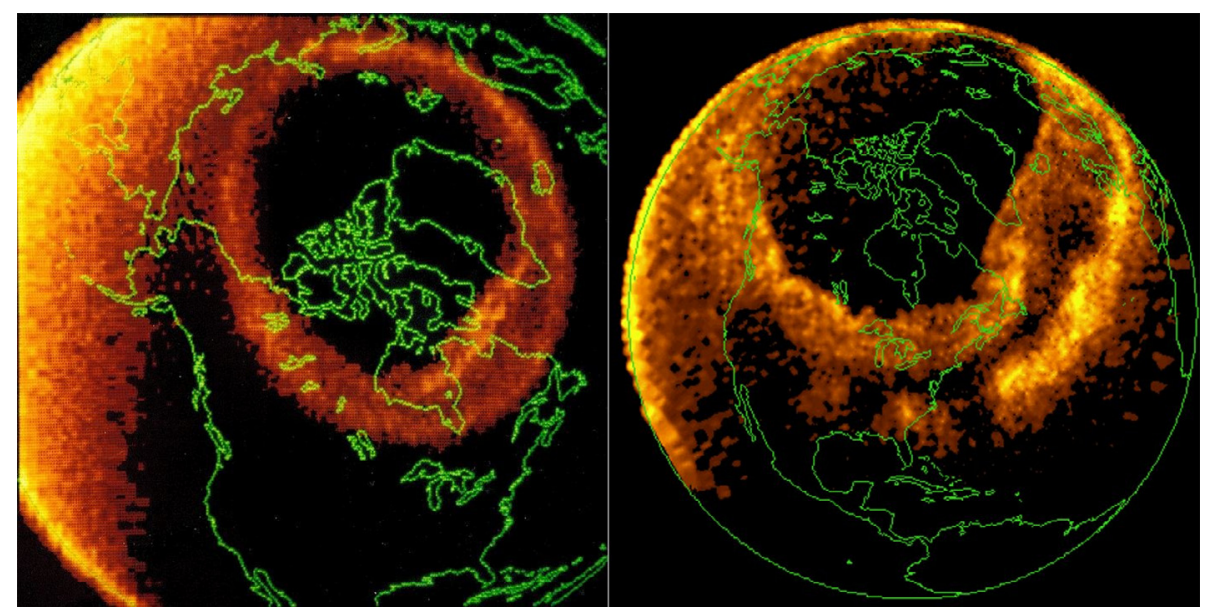

Fig. 1. The northern auroral oval as seen from the Dynamic Explorer 1 spin scan auroral imager under normal conditions (left). The bright light seen to the left represents scattered sunlight from the dayside of the Earth. The southern auroral oval as seen from the Dynamic Explorer 1 spin scan auroral imager (right) mapped to the northern hemisphere during the great storm of March 1989 (Source: NASA).

real-time solar wind data from the ACE spacecraft allowing for now- and forecasting. The Johns Hopkins University Applied Physics Laboratory OVATION site (http://sd-www.jhuapl. edu/Aurora/ovation_live/ovationdisplay.cgi?pole=N\&type=E), which uses real-time data from the DMSP satellites to determine the auroral oval. Similar to the latter is the auroral activity extracted from the POES satellites service, which is available from http://www.swpc.noaa.gov/pmap/index.html.

Sigernes et al. (2011) have recently developed a real-time nowcasting and forecasting service based on empirical models developed from optical data from ground all-sky cameras (Starkov 1994) and satellites (Zhang \& Paxton 2008). This service is available from the Kjell Henriksen Observatory web page (http://kho.unis.no/).

This paper describes the method for an "Online provision of auroral alert and tracking system", an auroral electrojet tracker, as designed in order to meet the requirements of Deliverable 4.3 in "WP-4 Forecasting tools and Modeling" of the European Union Frame Work program 7 AFFECTS (Advanced Forecast For Ensuring Communications Through Space) project. This auroral electrojet tracker, which is based on the assumption that the auroral electrojet is co-located with the auroral oval to a great extent, uses real-time measurements of the geomagnetic field variation to determine the equatorward and poleward edges of the auroral electrojet in the European sector. First, a short introduction about the auroral oval and how it is related to the auroral electrojets is given. A description of the methodology for finding the electrojet boundaries is described and simulations are performed to illustrate and verify the method. Next, a general description of the auroral electrojet tracker as provided for AFFECTS is given. Last the electrojet location, as determined by this method, in relation to auroral and precipitation boundaries and the possibilities of expanding and improving the current product, is discussed.

\section{Methodology}

2.1. Auroral oval determination by means of geomagnetic measurements - concept

The dynamics, sizes and shapes of the auroral ovals are determined by the interaction between the geomagnetic field and the

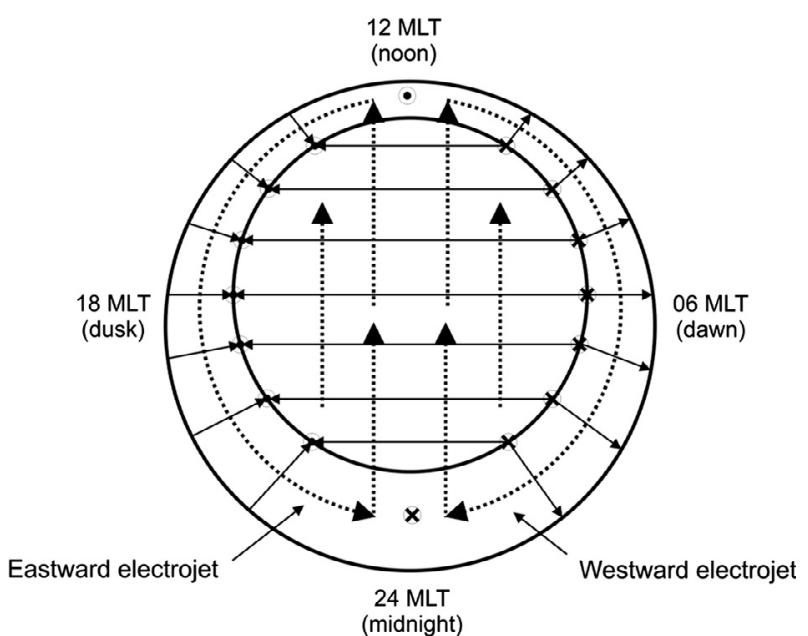

Fig. 2. Simplified relationship between auroral oval and electrojets. The auroral oval is represented by solid lines, Hall currents by dotted arrows and electrical fields by solid arrows.

solar wind. This interaction allows for currents flowing in and out of the upper atmosphere, and these currents are closed by horizontal currents in the ionosphere. Owing to channels of enhanced conductivity produced by particle precipitation, large Hall currents flow along the auroral ovals. These are termed the auroral electrojets. In the dusk sector in the northern hemisphere, the electrojet flows eastward, and in the dawn sector it flows westward. In Figure 2 the relationship between the electrojets and the auroral oval is illustrated in a simplified manner. In the figure the auroral oval is seen from above, the number 12 in the upper part indicates magnetic local noon and, thus, the direction towards the sun. Crosses and dots indicate currents flowing into and out of the ionosphere, solid arrows represent electrical fields. The dotted lines represent Hall currents induced by the presence of the electrical fields and the vertical geomagnetic field. As is seen, in the dusk sector (left part of the figure), the eastward electrojet is indicated within the oval. On the other side, in the dawn sector (right part of the figure), the westward electrojet is indicated. The magnetic field disturbances created by the auroral eletrojets are observed on a routine basis from 
any magnetometer station located in auroral latitudes. Since the electrojets are intimately connected to the auroral oval, and both are aligned in the east-west direction (along the lines of constant magnetic latitude), the latitudinal magnetic signature of the electrojet may be used in order to identify its position, and hence the position of the auroral oval. Furthermore, since the oval width and location is governed by the interaction between the geomagnetic field and the solar wind and therefore will change with time, its dynamic behaviour may be tracked using latitudinally spaced ground-based magnetometers.

\subsection{The latitudinal signature of the auroral electrojet}

As discussed above, the electrojets flow in the eastward and westward direction of the auroral oval in the dusk and dawn sectors, respectively. Any current in the ionosphere will induce its own magnetic field, and it is this field which is observed on a routine basis as magnetic field variation at stations at auroral latitudes. The latitudinal, magnetic signature of the electrojets may, as a first approximation, be simulated assuming they are sheet currents flowing $110 \mathrm{~km}\left(h_{0}\right)$ above ground. Assuming that a current sheet consists of latitude segments $(\mathrm{d} \lambda)$ of individual, straight line currents $(\sigma(\lambda) \mathrm{d} \lambda)$ with infinite length in the east-west direction, we may calculate the magnetic field contribution at a single observing latitude $\left(\lambda_{0}\right)$ from a single segment using Biot-Savart's law:

$$
B^{\prime}=\frac{\mu_{0}}{2 \pi} \cdot \frac{\sigma(\lambda) \mathrm{d} \lambda}{r}
$$

where $\lambda$ is the latitude of the current segment and $r$ is its distance from the observer $r(\lambda)=\left(R_{\mathrm{e}}^{2}+\left(R_{\mathrm{e}}+h_{0}\right)^{2}\right.$ $\left.-2 R_{\mathrm{e}}\left(R_{\mathrm{e}}+h_{0}\right) \cos \left(\lambda-\lambda_{0}\right)\right)^{1 / 2}$, where $R_{\mathrm{e}}$ is the Earth radius). The horizontal $(x-)$ and vertical $(z-)$ components at $\lambda_{0}$ are given as $B_{x}^{\prime}=B^{\prime} \sin (\alpha)$ and $B_{z}^{\prime}=B^{\prime} \cos (\alpha)$, where $\alpha$ is the elevation angle (from horizontal north) from $\lambda_{0}$ to the current segment $\left(\alpha(r)=90-a \sin \left(\frac{R_{\mathrm{e}}+h_{0}}{r} \cdot \sin \left(\lambda-\lambda_{0}\right)\right)\right)$. Having the contribution from a single current segment for both components, we may integrate over all segments from far to the south to far to the north of $\lambda_{0}$ in order to get the total magnetic field. Here we use a range of $\lambda_{0} \pm 10$ degrees:

$$
B_{x}\left(\lambda_{0}\right)=\int_{\lambda_{0}-10}^{\lambda_{0}+10} B_{x}^{\prime}
$$

and

$$
B_{z}\left(\lambda_{0}\right)=\int_{\lambda_{0}-10}^{\lambda_{0}+10} B_{z}^{\prime}
$$

The procedure can then be repeated for values of $\lambda_{0}$ with even spacing in the range of interest in order to produce a latitude profile for the magnetic field variation. This is illustrated for a situation in the auroral oval close to the dusk meridian in Figure 3, the situation corresponds to the location marked 18 to the left in Figure 2. The local coordinate system used in the simulations is the $x y z$ system commonly used in geomagnetic observations, where the $x$-axis points northwards, the $y$-axis points eastwards and the $z$-axis points downwards. In the bottom panel of Figure 3 the one-dimensional current density as a function of latitude $(\sigma(\lambda))$ is shown. At low latitudes the current is zero, between 65 and 70 degrees the eastward electrojet is flowing, hence the positive sign. North of the electrojet there is a weaker
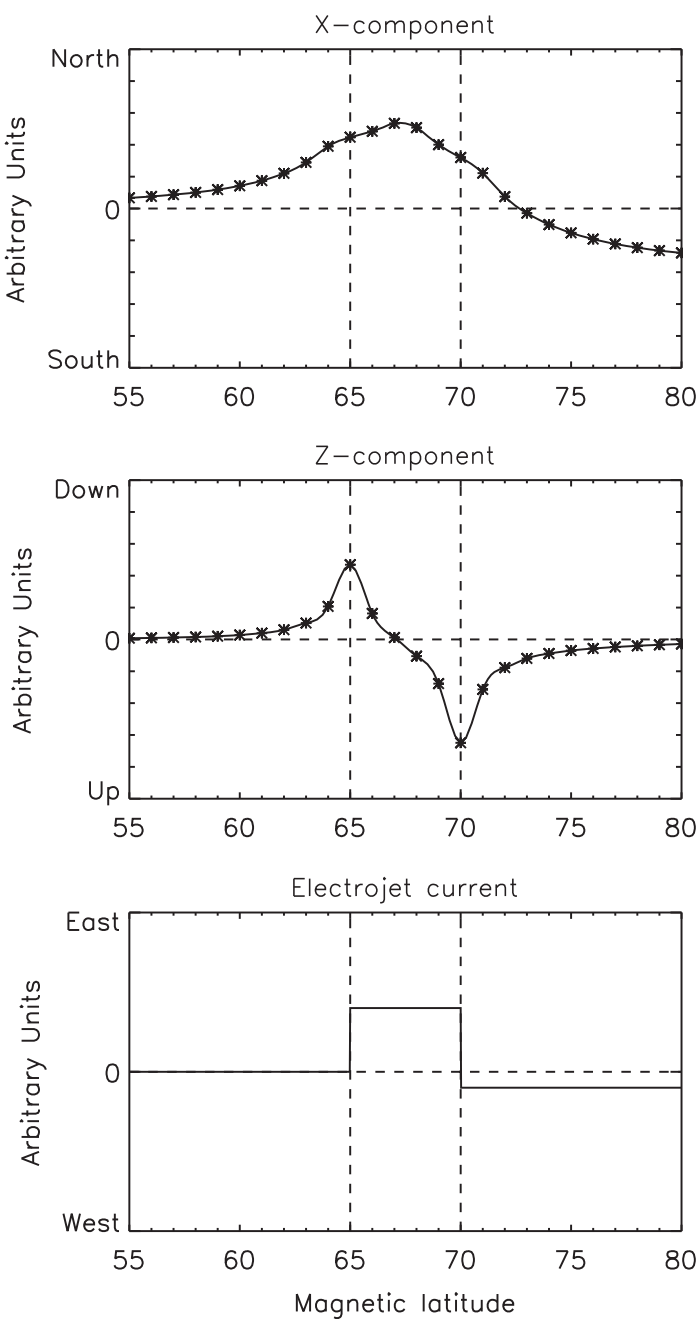

Fig. 3. Simulated magnetic field signature of eastward electrojet as function of magnetic latitude. Latitude profiles of horizontal $(x-)$ component (top) and vertical ( $z$-) component (middle) response of an eastward electrojet and weaker and oppositely directed polar cap current (bottom). Local $x y z$ coordinate system is used, where $x, y$ and $z$ points north, east and down, respectively.

polar cap return current flowing towards the sun/noon. In the top panel the resulting magnetic field variation $x$-component as function of latitude is shown, here the $x$-component being the horizontal component. As can be seen, the magnetic field increases to a maximum close to the middle of the electrojet, and it decreases below zero further north where the oppositely directed polar cap current is situated. It can also be seen that the $x$-component variation is dispersed latitudinally relative to the electrojet and thus cannot be used to accurately determine its extent. However, looking at the middle plot of Figure 3, we clearly see that the $z$-component (i.e., the vertical component) of the magnetic field variation gets a local maximum and minimum at the equatorward and poleward edges of the electrojet, respectively. This can be used to determine the location of the electrojet boundaries. In the case of a westward electrojet, the signs of the results in Figure 3 need to be changed, otherwise the result is the same. Hence, we summarize that in the dusk sector, the equatorward edge of the electrojet is represented by a maximum in the ground magnetic field variation $z$-component and the poleward edge is represented by a minimum. In the dawn sector it is opposite, the electrojet equatorward edge is represented by a minimum in the $z$-component and the 

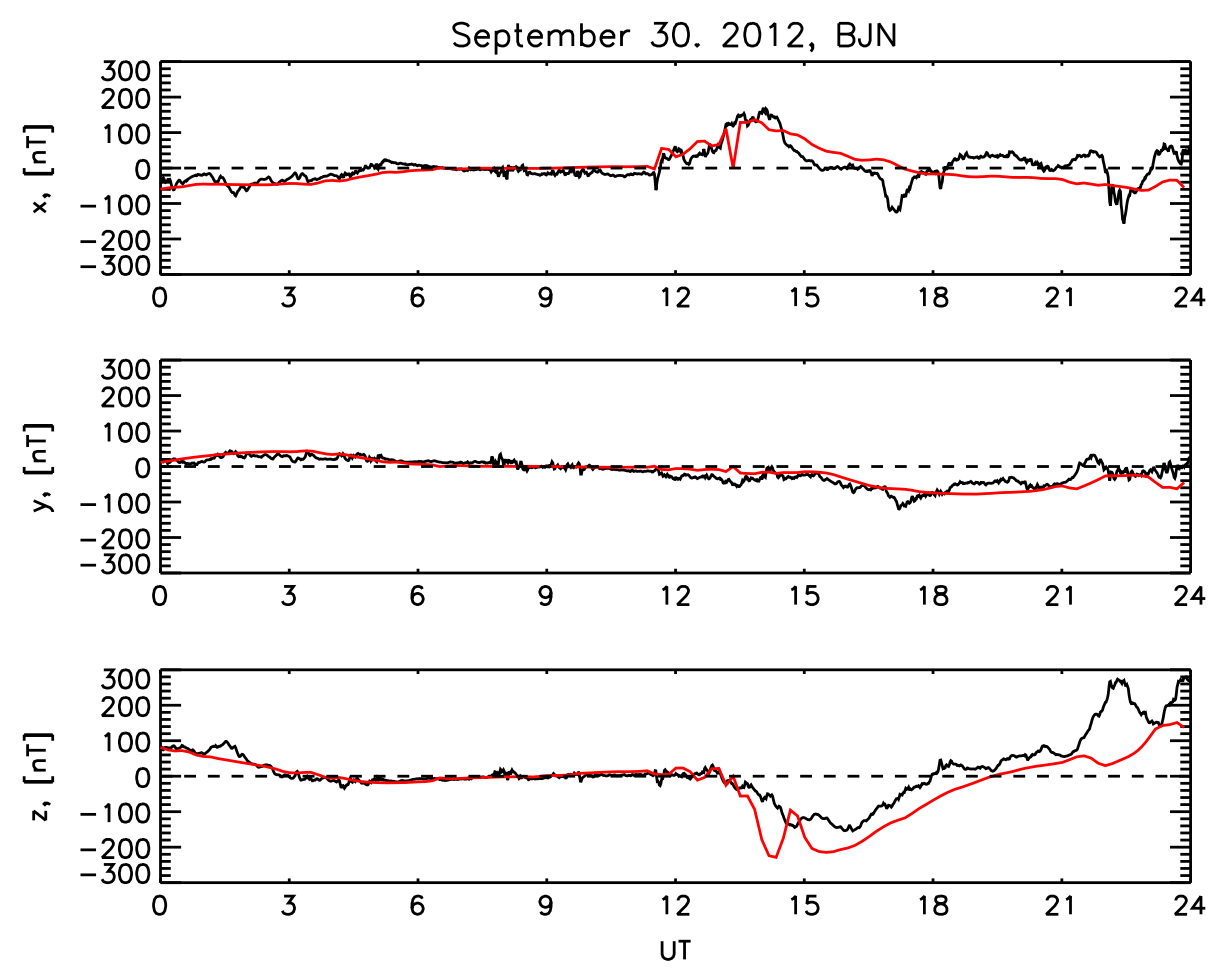

Fig. 4. Magnetogram for Bjornoya. Black curves are measured values. Red curves are modelled values.

poleward edge by a maximum. This method of obtaining the boundaries of the electrojet from latitudinal profiles of the vertical geomagnetic field variation was first applied by Kisabeth \& Rostoker (1971). They also thoroughly assessed and verified the method under a wide range of conditions using modelling (Kisabeth \& Rostoker 1971; Rostoker \& Kisabeth 1973).

\subsection{Auroral electrojet location obtained from a model}

In order to illustrate the method for obtaining the auroral electrojet boundaries, we have applied the method to the high latitude equivalent current system as derived from a model of the high latitude electric field and field aligned currents, the Weimer 2005 (W05) model (Weimer 2005a). From the W05 model it is possible to derive the ground magnetic field variation at a specific location and time owing to ionospheric currents quite successfully (Weimer 2005b). The model produces the high latitude ionospheric electric and magnetic potential patterns with the time, solar wind speed and density and interplanetary magnetic field $y$-and $z$-components as input. Using the method of Weimer (2005b) one may then from these potentials calculate the equivalent current in any given location:

$$
\mathbf{J}_{\mathbf{e}} \approx \hat{\mathbf{B}} \times \alpha\left(\nabla_{s} \psi \cdot \hat{\mathbf{E}}\right) \hat{\mathbf{E}}
$$

where $\mathbf{J}_{\mathbf{e}}$ is the equivalent current vector, $\hat{\mathbf{B}}$ is the local geomagnetic field direction (unity), $\alpha$ is the ratio between the Hall and Pedersen conductivity, $\psi$ is the magnetic potential owing to the field aligned currents and $\hat{\mathbf{E}}$ is the electric field direction (unity). Based on the discussion by Weimer (2005b) $\alpha$ was set to 1.5 , since this, according to him, provides the overall best results. Assuming a two-dimensional current sheet flowing in the ionosphere at an altitude of $110 \mathrm{~km}$, one may calculate the three-dimensional ground magnetic field variation $(\Delta \mathbf{B})$ from this current at a given location $\left(\mathbf{r}_{2}\right)$ using Biot-Savart integra- tion over an overhead area ( $2000 \mathrm{~km}$ wide) defined from the centre of the Earth by $\mathbf{r}_{1}$ :

$$
\Delta \mathbf{B}\left(\mathbf{r}_{2}\right)=\frac{\mu_{0}}{4 \pi} \int_{s} \frac{\mathbf{J}_{\mathrm{e}}\left(\mathbf{r}_{1}\right) \times\left(\mathbf{r}_{2}-\mathbf{r}_{1}\right)}{\left|\mathbf{r}_{2}-\mathbf{r}_{1}\right|^{3}} \mathrm{~d} A_{1} .
$$

In order to illustrate that we may use the W05 model in order to simulate real-world conditions, we show in Figure 4 a magnetogram for Bjørnøya (BJN) for one day (black curves) and the corresponding variations produced by the above-mentioned technique using time shifted ACE (OMNIweb) data as input for that particular day. As can be seen there is a good general agreement between the real and the modelled values, as was also found by Weimer (2005b). The W05 model does not reproduce substorms, and thus, the substorm electrojets seen occurring around UT 17.00 and 22.00 are not apparent in the modelled curves. Small differences between the modelled and measured results might also be attributed to the choice of a static ratio between Hall and Pedersen conductivities $(\alpha=1.5)$, although this does not change the overall good agreement between the curves.

Using the W05 we may now illustrate the concept discussed in Section 2.2 for a north-south chain of magnetometers in the European sector. The magnetometers used are summarized in Table 1. The magnetic field variation is calculated for the listed magnetometers and the local coordinate system is rotated so that the $x$-direction points towards the geomagnetic pole (rather than the geographic). Then the latitudinal variation profiles are created by fitting the modelled variations using natural splines. In Figure 5 we show the result for the same day as the magnetogram in Figure 4, September 30, 2012 UT 15.00. In the left panel we show a map of Northern Europe and Greenland. The magnetometers are indicated by red and blue dots, and arrows originating from the dots indicate the local horizontal magnetic field variation rotated $90^{\circ}$ counter-clockwise 
M.G. Johnsen: Real-time determination and monitoring of the auroral electrojet boundaries

Table 1. List of magnetometers used in this work. Magnetic latitudes and midnight times were acquired through the corrected geomagnetic (CGM) coordinate system service at http://omniweb.gsfc.nasa.gov/vitmo/cgm_vitmo.html (January 2013).

\begin{tabular}{|c|c|c|c|c|}
\hline \multirow[b]{2}{*}{ Station } & \multicolumn{2}{|c|}{ Geographic } & \multicolumn{2}{|c|}{ Magnetic (CGM 2012, $100 \mathrm{~km}$ ) } \\
\hline & Latitude $\left({ }^{\circ}\right)$ & Longitude $\left(^{\circ}\right)$ & Latitude $\left({ }^{\circ}\right)$ & Midnight (UTC) \\
\hline Ny-Ålesund (NAL) & 78.92 & 11.93 & 76.45 & 21.04 \\
\hline Longyearbyen (LYR) & 78.20 & 15.83 & 75.45 & 20.59 \\
\hline Hopen (HOP) & 76.51 & 25.01 & 73.36 & 20.44 \\
\hline Bjørnøya (BJN) & 74.50 & 19.00 & 71.68 & 21.12 \\
\hline Sørøya (SOR) & 70.54 & 22.22 & 67.55 & 21.17 \\
\hline Andenes (AND) & 69.30 & 16.03 & 66.59 & 21.41 \\
\hline Dønna (DON) & 66.11 & 12.50 & 63.45 & 22.02 \\
\hline Rørvik (ROR) & 64.95 & 10.99 & 62.30 & 22.10 \\
\hline Dombås (DOB) & 62.07 & 9.11 & 59.30 & 22.23 \\
\hline Solund (SOL) & 61.08 & 4.84 & 58.46 & 22.41 \\
\hline Karmøy (KAR) & 59.21 & 5.24 & 56.36 & 22.43 \\
\hline
\end{tabular}


Data time stamp: $09 / 30 / 2012 \quad 15: 00$

Fig. 5. Acquired auroral oval boundaries in the dusk sector using magnetic field variations produced from the W05 electric potential and field aligned current model. The map (left) shows the electric potential pattern, calculated disturbance vectors at selected magnetometer locations and determined electrojet boundaries (red and green). The direction towards the sun is indicated with the black vector above the map. Time of the model run is stated above the map, and time stamp for simulated conditions in the lower right corner. The latitudinal profile of the vertical (z-) component is presented in the upper right plot, the solid line represents a natural spline fit to the simulated measurements (crosses). Vertical green and red lines indicate obtained equatorward and poleward electrojet boundaries, respectively. The latitude profile of the horizontal (solid line) and $x$ - (dashed line) components is presented in the lower right plot. Crosses indicate simulated measurements. Stars in both plots indicate simulated measurements $(z$ and $x$ ) from a thought magnetometer chain located strictly along the magnetic meridian.

(i.e., the magnetic equivalent convection, MEC). The calculated high latitude electric potential pattern is indicated as isocontours, here the European sector is in the dusk (evening) convection cell. Closely spaced isocontours indicate large gradients in the electric potential which corresponds to large $E$-fields that give rise to strong currents. In the right panels we show the calculated magnetic field variation $z$ - (top), $x$ - and $H$ - (bottom) components as function of magnetic latitude. In the top plot values at the magnetometers are indicated as crosses, stars indicate the profile if the magnetometers had been situated strictly along one magnetic meridian going from Svalbard and south through Finland. In the bottom plot red crosses indicate the $x$-component while black crosses indicate the horizontal component $\left(\sqrt{x^{2}+y^{2}}\right)$. The dashed line represents the latitudinal profile of the $x$-variation. The $x$-component being positive with maximum near $69^{\circ}$ indicates that we here are observing within the eastward electrojet. The change of sign to the north indicates the existence of a sunward polar cap current. In the dawn sector the $x$-component would show a minimum (rather than a maximum) in the vicinity of the electrojet owing to its opposite direction. Using the method as described in Section 2.2, in the top right plot the electrojet equatorward and poleward boundaries have been identified by the maximum (green line) and minimum (red line), respectively. The boundaries are indicated by dashed lines with the same colours on the map. It is clearly seen that the identified electrojet boundaries cover the electrojet as indicated by the electric potentials represented by isocontours. Comparing the latitude profile from the magnetometers with that strictly following the meridian (top, right plot), it also becomes evident that the exact alignment of the magnetometers along the magnetic meridian is not crucial for the boundary determination. 


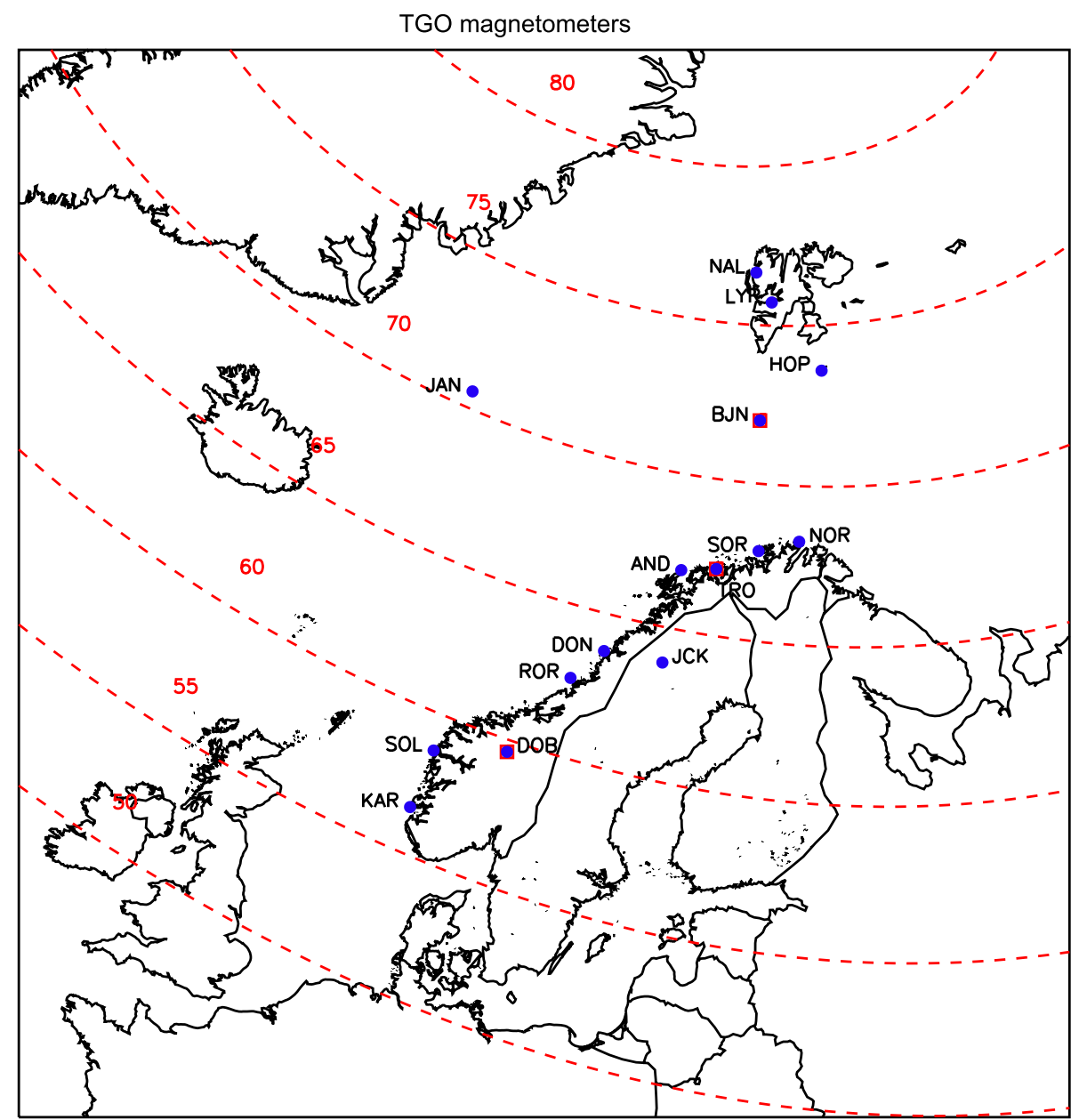

Fig. 6. Map showing magnetometers operated by TGO (blue dots). The magnetometer at Jan Mayen (JAN) is currently not in operation.

\section{Online provision of a real-time auroral alert and tracking system}

\subsection{Tromsø Geophysical Observatory - geomagnetic measurements}

One of the main duties of Tromsø Geophysical Observatory (TGO) is to maintain geomagnetic measurements in mainland Norway and the Arctic Ocean around Svalbard. Currently TGO operates 14 flux-gate magnetometers evenly spaced covering geographic latitudes from $59^{\circ} \mathrm{N}$ to $79^{\circ} \mathrm{N}$. The locations of the TGO magnetometers are indicated as blue dots on the map in Figure 6. TGO receives data from all magnetometers in near real-time, with the longest delay being $2 \mathrm{~min}$. All data are available from http://www.tgo.uit.no/ (January 2013) with both $10 \mathrm{~s}$ resolution and postprocessed $1 \mathrm{~min}$ resolution. The TGO magnetometer network is evenly spaced in latitude in a north-south manner, which makes its measured data an excellent starting point for the development of a real-time auroral electrojet alert and tracking system for the European sector.

\subsection{Auroral tracking and alert system based on TGO magnetometer data}

Based on the theoretical foundation drawn in Section 2, as part of the EU Framework Program 7 project AFFECTS (http://www.affects-fp7.eu, January 2013), an auroral electrojet tracker based on data from the TGO magnetometer network has been developed. The tracker consists of a numerical and two graphical parts. The graphical parts are displayed together with a short description at the following URL: http://fox.phys.uit.no/ AFFECTS/ (January 2013).

The first graphical part is a map where the poleward and equatorward edges of the auroral oval, determined from the magnetic field variation $z$-component as described in Section 2, are indicated as green and red dashed lines. In addition plots where the relevant components of the geomagnetic field variations as function of latitude are displayed. The time of the last oval determination is indicated on top of the map, and the corresponding time stamp for the data used is indicated in the bottom right corner. The direction towards the sun is indicated by an arrow at the top of the map.

The second graphical part is a graphical representation of the strength and latitude of the maximum in horizontal geomagnetic variations in the European sector, it may be referred to as an activity indicator. The activity is indicated by a bar updating in real-time according to the maximum magnetic field variation ( $x$-component) found in the data. The scale chosen to indicate the activity corresponds in the interval $0-8$ linearly to $0-1500 \mathrm{nT}$ and in the interval $8-10$ to $1500-3000 \mathrm{nT}$. As a qualitative guide to the level of activity, values that correspond approximately to quiet, moderate, active and extraordinary 
Run: Mon Jun 24 12:17:38 2013



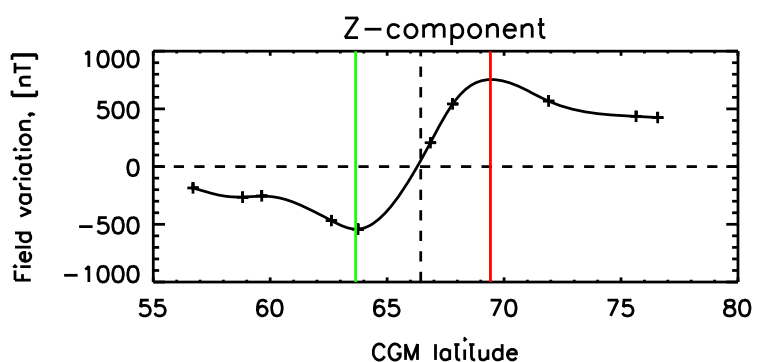

CGM Ialilude

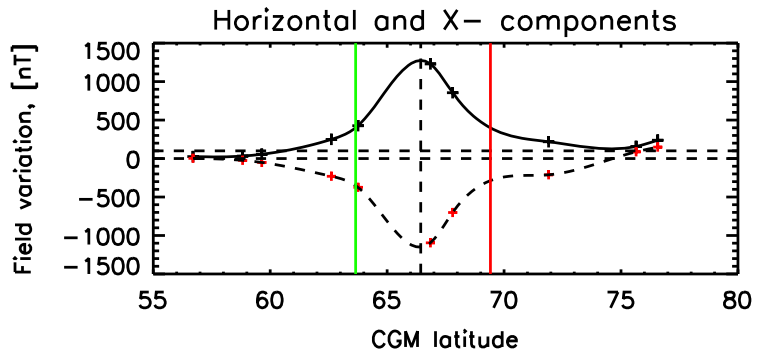

Data time stamp: 18/06/2012 03:00:00

Fig. 7. Acquired auroral oval boundaries in dawn sector using the AFFECTS auroral electrojet tracker.

Auroral disturbance



Latitude of Maximum activity

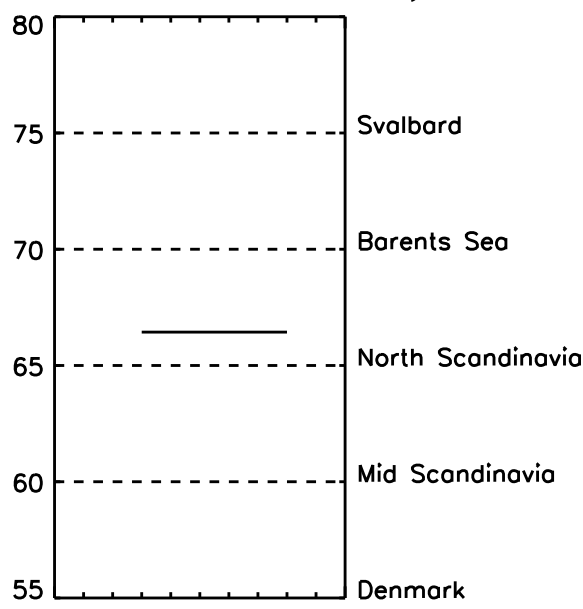

Fig. 8. Activity indicator for the same event as presented in Figure 7.

conditions are marked. The bar is accompanied with another display indicating the latitude of the maximum disturbance.

The numerical part is an ascii file, which is updated in near real-time, containing values of the poleward and equatorward edges of the auroral oval at present and the previous three hours. The ascii file can be found on the following URL: http://fox.phys.uit.no/AFFECTS/RT oval location.dat (January 2013). For the electrojet tracker, data from nine of TGO's 14 magnetometers have been used in order to get as even latitudinal spacing between them as possible (see Table 1). Owing to the uncertainties in quiet-time determination of the measured magnetic field as well as the variations associated with the solar quiet mean $(\mathrm{Sq})$ current system, it has proved difficult to get meaningful latitudinal profiles of the magnetic field variations under very quiet conditions. Therefore, the auroral electrojet determination is turned off when the maximum in the absolute value of the $x$-component reaches values of less than $75 \mathrm{nT}$. This is displayed as a message in the graphical display and as $\mathrm{NaN}$ (not a number) in the ascii file. Owing to the greater complexity of the current systems near magnetic noon (cusp,
DPY current) (e.g., Vennerstrom et al. 1984) and around midnight (Harang discontinuity) (e.g., Harang 1946a, 1946b; Heppner 1972), the oval determination from the $z$-component is turned off in the intervals UT 07.00-11.00 and UT 19.30-00.30. However, in order to indicate where the oval is located and the degree of activity, the maximum in the $x$-component and latitude for it, is still found during these intervals (i.e., second graphic display).

In Figure 7 the obtained electrojet edges from the magnetic signature of the westward electrojet in the dawn sector are presented in the same fashion as in the graphical display on the web page of the tracker and in Figure 5. Comparing with the simulations in Figures 3 and 5, it is seen that the signature of the electrojet as obtained from the TGO magnetometers is very similar. In Figure 8 the activity indicator, which is the second graphical display of the auroral electrojet tracker, is shown for the same event as displayed in Figure 7. As can be seen the activity is rather high and situated in northern Fennoscandia. This is also reflected in the MEC vectors in Figure 7. Another example, but this time from the eastward electrojet in the dusk 
Run: Mon Jun 24 12:15:26 2013

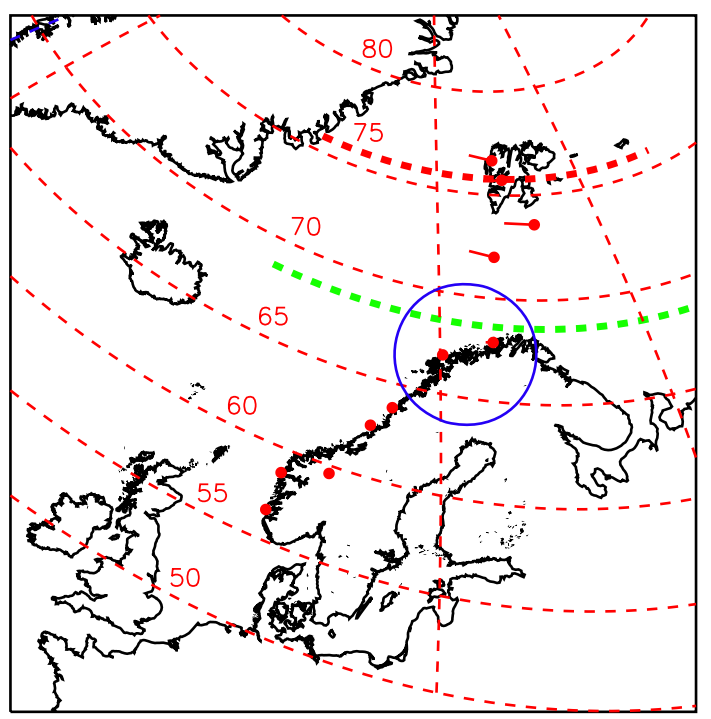

Z-component

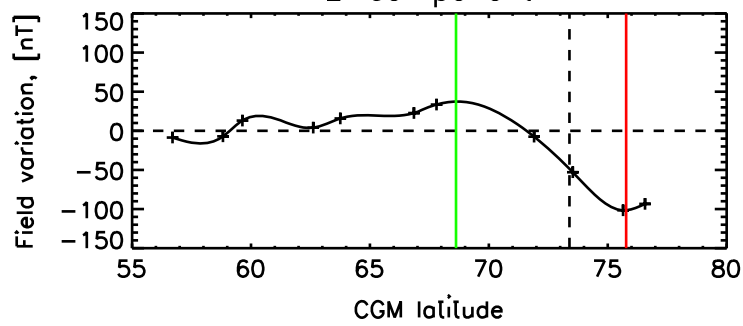

Horizontal and $\mathrm{X}$ - components



Dota time stamp: 15/06/2012 15:00:00

Fig. 9. Acquired auroral oval boundaries in dusk sector using the AFFECTS auroral electrojet tracker.

Auroral disturbance

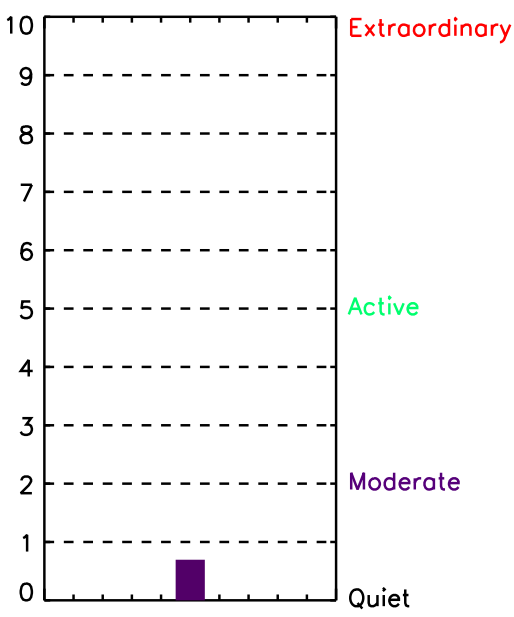

Latitude of Maximum activity

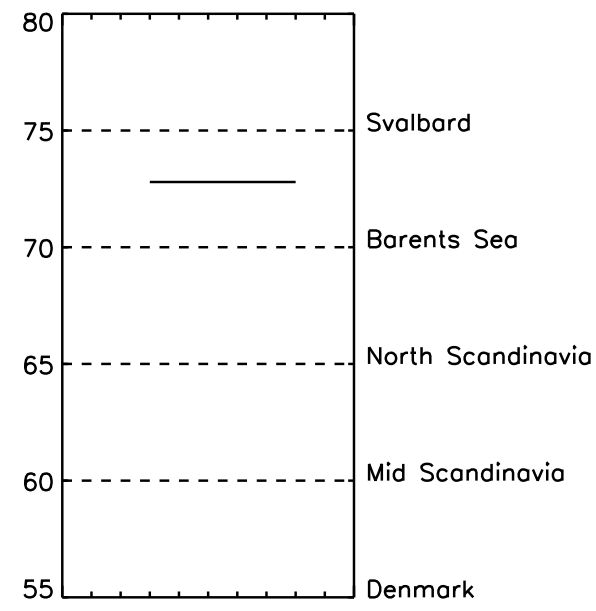

Fig. 10. Activity indicator for the same event as presented in Figure 9.

sector, is shown in Figure 9. Here the electrojet is situated further north, but the magnetic field signature as defined by Figure 3 is very clear. In Figure 10 the activity indicator for the same case is shown, and as can be seen the activity is rather quiet and located over the Barents sea close to Svalbard.

\section{Discussion and concluding remarks}

We have in this paper described a method for obtaining the poleward and equatorward boundaries of the auroral electrojets from a meridian chain of magnetometers. For illustrative purposes we have shown by applying an ionospheric electric potential and field aligned current model, how we may reproduce the ground magnetic field variation caused by large-scale magnetosphere ionosphere interaction. This has, furthermore, been used to illustrate how the electrojet as determined by the meridian chain method on data produced by the model, covers the sunward convection in the high latitude convection pattern as provided by the same model.
The main assumption for the determination of the electrojet based on data from a magnetometer chain and to use its location as a proxy for the auroral oval, is that the electrojet is coincident with the auroral oval. From a qualitative point of view this is a reasonable assumption. Particle precipitation, that causes the aurora, also increases the degree of ionization in the ionosphere, and thereby also increases the conductivity. Thus, there will be a channel of increased conductivity collocated with the auroral oval. The increased conductivity implies increased currents if electric fields are present, in the case of the electrojets the high latitude convection electric field.

Several studies have been performed to examine the relationship between the electrojet as determined from a magnetometer chain, and the auroral oval. Of particular interest are studies by Walker (1964), Wallis et al. (1976), Winningham et al. (1979) and Rostoker et al. (1979), where all-sky cameras, satellite-based scanning photometers and particle detectors were used in order to determine the auroral boundaries. From these studies we may conclude that the electrojet is coincident with 
the diffuse auroral oval. In the evening quadrant the eastward electrojet will be entirely within the auroral oval which will sometimes extend somewhat further north. In the afternoon quadrant on the other hand, the electrojet will appear wider than the oval owing to enhanced conductivities on sub auroral latitudes. We should note here that this latter effect will have a seasonal variation since the amount of absorbed UV light above a given location depends on the solar zenith angle. Also, the study by Rostoker et al. (1979), where this was found, was performed in the Canadian sector, which is where the auroral oval reaches its lowest geographical latitudes. In the European sector the auroral oval is much further north, and thus, although one may expect the effect to be there during summer, it might be considerably weaker or non-existent during winter.

All the studies mentioned above were performed in the dusk sector. We are not aware of any similar comparisons between the westward electrojet and the auroral oval in the dawn sector. However, as is also stated by Wallis et al. (1976), there is no evidence that the westward electrojet exhibits different behaviour from the eastward electrojet. We may thus, assume that the determination of the electrojet in the dawn sector may be used as a proxy for the location of the auroral oval just as well as in the dusk sector.

It is important to point out that the electrojets and the aurora are manifestations of the same interaction between the solar wind and the magnetosphere-ionosphere system, and they will react in a similar fashion to severe space weather events. As for space weather and monitoring of the ionospheric conditions, although there may exist differences between the location of the electrojet and optical phenomenon, monitoring the former may in fact be more useful than monitoring the latter. After all, it is the currents in the electrojets representing the larger geomagnetic disturbance, that pose challenges towards ground-based, modern infrastructure and technology such as navigation and power transmission.

We have furthermore, in this paper, presented an "auroral electrojet tracker", provided for the AFFECTS space weather project, where the electrojet is determined in near real-time based on data from the TGO magnetometer chain. It may be found at http://fox.phys.uit.no/AFFECTS/ (January 2013). The TGO magnetometer chain may not be ideal for electrojet determination since it is not strictly aligned with the geomagnetic meridian. However, we have shown in this paper that this is not a severe problem and not strictly necessary. As far as we know, there are currently no other techniques than the one used here for doing continuous and real-time monitoring of the location of the electrojet/auroral oval. All other existing auroral tracking and nowcasting concepts are based on data from point measurements that are made regularly, which then are fed into empirical models. With real-time data from a ground-based magnetometer chain, the use of empirical models, which are statistical in their nature, is not necessary.

In the future it will be desirable to use other chains in addition to the TGO-chain for multipoint determination of the auroral electrojets. Good magnetometer chains and networks where the problems of real-time data handling have been overcome, such as IMAGE, the Greenland magnetometer chain, CARISMA and GIMA, already exist. With agreements of mutual exchange of real-time data, it will be possible for anyone to provide almost circumpolar real-time determinations of the location of the electrojets and the geomagnetic activity, which would be very desirable from a space weather point of view and very beneficial for the community as a whole. The resurrection of similar magnetometer chains in Russia would complete and improve this picture further. Using the electrojet technique on historic data from all these magnetometer chains could provide empirical models for the electrojet/auroral oval which could then be used for forecasting purposes.

Acknowledgements. Daniel R. Weimer is thanked for developing the Weimer 2005 empirical model and making it available. This study received the Weimer 2005 model from the CEDAR Database at the National Center for Atmospheric Research, which is supported by the National Science Foundation of the United States of America. The OMNI data were obtained from the GSFC/SPDF OMNIWeb interface at http://omniweb.gsfc.nasa.gov. We thank the ACE MAG and SWEPAM instrument teams and the ACE Science Center for providing the ACE data. The research leading to some of these results has received funding from the European Commission's Seventh Framework Programme (FP7/2007-2013) under the Grant Agreement No. 263506 (AFFECTS). The author would like to thank the reviewers for their useful comments and helping to make this a better paper.

\section{References}

Elphinstone, R.D., K. Jankowska, J.S. Murphree, and L.L. Cogger, The configuration of the auroral distribution for interplanetary magnetic field Bz northward. I - IMF Bx and By dependencies as observed by the Viking satellite, J. Geophys. Res., 95, 5791-5804, 1990.

Harang, L., The mean field of the polar earth-magnetic storm, Geofysiske Publikasjoner (Geophysica Norwegica), 16, 1-44, $1946 \mathrm{a}$.

Harang, L., The mean field of disturbance of polar geomagnetic storms, Terrestrial Magnetism and Atmospheric Electricity (J. Geophys. Res.), 51, 353, 1946 b.

Heppner, J.P., The Harang discontinuity in auroral belt ionospheric currents, Geofysiske Publikasjoner (Geophysica Norwegica), 29, 105-120, 1972

Kabin, K., R. Rankin, G. Rostoker, R. Marchand, I.J. Rae, A.J. Ridley, T.I. Gombosi, C.R. Clauer, and D.L. DeZeeuw, Openclosed field line boundary position: a parametric study using an MHD model, J. Geophys. Res. (Space Phys.), 109, A05222, 2004.

Kisabeth, J.L., and G. Rostoker, Development of the polar electrojet during polar magnetic substorms, J. Geophys. Res., 76, 6815, 1971.

Machol, J.L., J.C. Green, R.J. Redmon, R.A. Viereck, and P.T. Newell, Evaluation of OVATION Prime as a forecast model for visible aurorae, Space Weather, 10, S03005, 2012.

Milan, S.E., M. Lester, S.W.H. Cowley, K. Oksavik, M. Brittnacher, R.A. Greenwald, G. Sofko, and J.-P. Villain, Variations in the polar cap area during two substorm cycles, Ann. Geophys., 21, 1121-1140, 2003.

Milan, S.E., G. Provan, and B. Hubert, Magnetic flux transport in the Dungey cycle: a survey of dayside and nightside reconnection rates, J. Geophys. Res., 112, A01209, 2007.

Newell, P.T., T. Sotirelis, J.M. Ruohoniemi, J.F. Carbary, K. Liou, J.P. Skura, C.-I. Meng, C. Deehr, D. Wilkinson, and F.J. Rich, OVATION: oval variation, assessment, tracking, intensity, and online nowcasting, Ann. Geophys., 20, 1039-1047, 2002.

Newell, P.T., T. Sotirelis, K. Liou, A.R. Lee, S. Wing, J. Green, and R. Redmon, Predictive ability of four auroral precipitation models as evaluated using Polar UVI global images, Space Weather, 8 , S12004, 2010a.

Newell, P.T., T. Sotirelis, and S. Wing, Seasonal variations in diffuse, monoenergetic, and broadband aurora, J. Geophys. Res. (Space Phys.), 115, A03216, 2010b.

Rae, I.J., K. Kabin, J.Y. Lu, R. Rankin, S.E. Milan, F.R. Fenrich, C.E.J. Watt, J.-C. Zhang, A.J. Ridley, T.I. Gombosi, C.R. Clauer, G. Tóth, and D.L. DeZeeuw, Comparison of the open-closed separatrix in a global magnetospheric simulation with observations: the role of the ring current, J. Geophys. Res. (Space Phys.), 115, A08216, 2010. 
Rostoker, G., and J.L. Kisabeth, Response of the polar electrojets in the evening sector to polar magnetic substorms, J. Geophys. Res., 78, 5559, 1973.

Rostoker, G., K. Kawasaki, T.J. Hughes, J.D. Winningham, and J.R. Burrows, Energetic particle precipitation into the high-latitude ionosphere and the auroral electrojets. II - Eastward electrojet and field-aligned current flow at the dusk meridian, J. Geophys. Res., 84, 2006-2018, 1979.

Sigernes, F., M. Dyrland, P. Brekke, S. Chernouss, D.A. Lorentzen, K. Oksavik, and C. Sterling Deehr, Two methods to forecast auroral displays, J. Space Weather Space Clim., 1, A03, 2011.

Starkov, G., Mathematical model of the auroral boundaries, Geomag. Aeron., 34, 331-336, 1994.

Vennerstrom, S., E. Friis-Christensen, T.S. Jorgensen, O. Rasmussen, C.R. Clauer, and V.B. Wickwar, Ionospheric currents and F-region plasma boundaries near the dayside cusp, Geophys. Res. Lett., 11, 903906, 1984.

Walker, J.K., Space-time associations of the aurora and magnetic disturbance, J. Atmos. Terr. Phys., 26, 951-954, 1964.
Wallis, D.D., G. Rostoker, and C.D. Anger, The spatial relationship of auroral electrojets and visible aurora in the evening sector, J. Geophys. Res., 81, 2857-2869, 1976.

Weimer, D.R., Improved ionospheric electrodynamic models and application to calculating Joule heating rates, J. Geophys. Res. (Space Phys.), 110, A05306, 2005a.

Weimer, D.R., Predicting surface geomagnetic variations using ionospheric electrodynamic models, J. Geophys. Res. (Space Phys.), 110, A12307, 2005b.

Winningham, J.D., K. Kawasaki, and G. Rostoker, Energetic particle precipitation into the high-latitude ionosphere and the auroral electrojets. I - Definition of electrojet boundaries using energetic electron spectra and ground-based magnetometer data, J. Geophys. Res., 84, 1993-2005, 1979.

Zhang, Y., and L.J. Paxton, An empirical Kp-dependent global auroral model based on TIMED/GUVIFUV data, J. Atmos. Sol. Terr. Phys., 70, 1231-1242, 2008.

Cite this article as: Johnsen MG: Real-time determination and monitoring of the auroral electrojet boundaries. J. Space Weather Space Clim., 2013, 3, A28. 